\title{
Reproductive and menstrual history of females with fragile $X$ expansions
}

\author{
Anna Murray ${ }^{1}$, Sarah Ennis ${ }^{2}$, Felicity MacSwiney ${ }^{1}$, James Webb ${ }^{1}$ and Newton E Morton ${ }^{2}$ \\ ${ }^{1}$ Wessex Regional Genetics Laboratory, Salisbury District Hospital, Wiltshire; ${ }^{2}$ Human Genetics Research Division, \\ Southampton University Hospitals Trust, Southampton, UK
}

FRAXA premutations have been associated with premature ovarian failure (POF) or menopause before the age of 40 . We have studied women in families ascertained because of a mentally retarded full mutation relative and determined their age of menopause, serum hormone levels in premenopausal individuals and the outcome of any pregnancies. Survival analysis was used as a measure of menopause and demonstrated a significant decrease in age of menopause in premutation carriers compared with their full mutation carrier and normal relatives. Serum FSH was also raised in premutation carriers, although oestradiol, inhibin A and inhibin B were not significantly different. However, we did not find an excess of dizygous twins or pregnancy loss/trisomies, both of which are associated with aging ovaries. Thus premutation carriers as a group have an earlier menopause and raised serum FSH but do not appear to manifest other features of an aging ovary. European Journal of Human Genetics (2000) 8, 247-252.

Keywords: Fragile X; premutation; premature ovarian failure

\section{Introduction}

FMR1 is a member of a group of genes which give rise to a disease phenotype by dynamic mutation of a trinucleotide repeat. ${ }^{1}$ While the trinucleotide repeat disease genes have common features, there are a number of notable differences. Expansions in FMR1 are associated with the fragile $X$ syndrome, but only when the gene is methylated, and this occurs when the number of CGG repeats exceeds about 200. Further expansion of the repeat does not generate a more severe phenotype, and in this respect fragile $X$ differs from most trinucleotide repeat diseases. The phen otype characteristically associated with FM R1 expansion is moderate mental retardation and in addition there may be various physical and behavioural features.

FMR1 has a premutation category, with unmethylated expansions of approximately 50-200 repeats. ${ }^{2,3}$ Premutations were thought to be without phenotypic effect, supported by molecular evidence that FMR1 protein is identical whether transcribed from a normal or premutation allele. ${ }^{4}$ However,

Correspondence: Anna Murray, Wessex Regional Genetics Laboratory, Salisbury District Hospital, Salisbury, Wiltshire, SP2 8B], UK.

Tel: +44 1722 425048; Fax: +441722338095

Received 28 September 1999; revised 19 November 1999; accepted

24 November 1999 there is evidence that female premutation carriers are at increased risk of premature ovarian failure (POF) or menopause before the age of $40 .^{5,6}$

An international collaboration involving over 700 women from fragile $X$ families concluded that premutation carriers over the age of 40 had approximately a $24 \%$ chance of having POF compared with $<1 \%$ of normal relatives and full mutation carriers. ${ }^{6}$ Also studies of women ascertained because of POF have shown that they have about a $2 \%$ chance of being a premutation carrier, which can rise to $16 \%$ for familial cases. ${ }^{7}$ A study by Turner et $\mathrm{al}^{8}$ suggested an increased incidence of dizygous twinning in premutation carriers but not in full mutations and normal relatives, but this was not substantiated in a study by Sherman et al. ${ }^{9}$ The rate of dizygous twinning is known to increase with maternal age, ${ }^{10}$ and an excess of dizygous twins in premutation carriers might be symptomatic of the general ised premature aging of the ovaries that gives rise to early menopause. Inference might predict that premutation carriers have an excess of trisomic conceptuses, as this too is correlated with advanced maternal age.

The aim of the present study was to determine whether all premutation carriers tend to have early menopause or alternatively whether those without POF have a normal distribution of menopause age, and also to determine the 
frequency of twinning and trisomies in premutation carriers.

\section{Materials and methods \\ Study population}

Individuals were selected from families ascertained through a mentally retarded proband with the fragile $X$ syndrome (FRAXA), in whom we knew the fragile $X$ status prior to the study. In all, 352 women were included: 31 with full mutations (methylated expansion), 116 with premutations (51-200 repeats and unmethylated) and 205 first and second degree relatives of the mutation carriers with FRAXA repeats of 50 and under.

\section{Protocol}

Women were contacted by telephone, and a home visit arranged, lasting about an hour. During the home visit women were questioned about their reproductive and menstrual histories. In a minority of cases individuals had moved out of the area, and these women were interviewed over the telephone. All women who were still menstruating were asked to provide a blood sample for hormone analysis on days $3-5$ of their cycle. This was usually achieved by asking them to visit their general practitioners on the appropriate day. Clinically significant hormone results were relayed to the patient.

\section{Hormone analysis}

Serum was separated from a $10 \mathrm{ml}$ blood sample, stored at $-20^{\circ} \mathrm{C}$ and batches were sent to the testing laboratories. FSH and oestradiol were tested at the Middlesex Hospital, London, using standard techniques, and inhibin $A$ and $B$ at the Centre for Reproductive Biology, Edinburgh, using the techniques of Groome et al. ${ }^{11,12}$

\section{Statistical analysis}

Information from patient interviews was coded and entered into an ACCESS database for statistical analysis. Survival analysis was used to determine the effect of repeat size on menopause using the Lifetest Procedure (SAS Institute Inc). For women who had not yet experienced menopause, their age at interview was taken as a censored value. For women who had an unnatural menopause, usually hysterectomy, their age at hysterectomy was taken as a censored value. For women who had al ready experienced menopause, their age at menopause was taken and not censored. All other analyses were by SAS regression analysis. The results quoted are all linear, but quadratic and stepwise regressions were also carried out.

\section{Results}

\section{Incidence of POF}

POF was defined as natural menopause before the age of 40 and 14 of the 352 women interviewed had POF. However, the distribution was not random between the size categories: there were three in those with normal alleles (1.5\%), 10 in premutations (8.6\%) and two in full mutations (6.5\%). If only women over age 40 at the time of interview were considered, $2 / 92$ normals $(2.2 \%), \quad 10 / 66$ premutations (15.2\%) and $2 / 11$ full mutations (18.2\%) had POF. Therefore although the difference between the incidence of POF is significant for premutations vs normals, the number of full mutations is too small to draw significant conclusions. However, these data were submitted to the international collaborative study which demonstrated that full mutation carriers are not at a significant risk for POF. The two full mutation carriers in our series with POF were from the same family, and so possibly coincidental familial POF cases, unrelated to fragile $X$ status.

\section{Survival analysis}

Pairwise comparisons were made, but there was no difference between the full mutation and control groups; they were therefore combined and compared with the premutation group (Figure1). There was a significant shift in menopause age in the premutation group, with a median of 49 compared with 54 in the others. The log rank test gave a $X_{1}^{2}$ of 17.39 $(P=0.0001)$. The mean menopause age in the premutation group was 47.87 years $\left(\mathrm{SE}_{\text {mean }}=0.8825\right)$ compared to 52.96 years in the controls $\left(\mathrm{SE}_{\text {mean }}=0.7733\right)$.

\section{Size of premutation and $X$ inactivation ratio vs age of menopause}

A second survival analysis was performed including FRAXA repeat size, but this did not add to the significance. There was also no significant correlation between repeat number and age of menopause in the premutation category alone $\left(\mathrm{X}_{1}^{2}=0.0015, \mathrm{P}=0.97\right)$ (Figure2). A possible modifier of a premutation effect is $X$ inactivation skewing, but there was no correlation between the age of menopause and the percentage of active $\mathrm{X}$ chromosomes that carried the premutation (Figure 3 ).

\section{Frequency of chromosome abnormalities and twins}

Each woman was asked to give details of all conceptuses, to compare the frequency of twinning and trisomies. Livebirths, stillbirths, spontaneous abortions and therapeutic abortions (not including those for fragile $X$ full mutation foetuses) were included and elective abortions, ectopic pregnancies and unknowns were excluded, giving a total of 555 records. The probability of zygosity was estimated for each set of 'unknown' twins. There was no correlation between repeat size and either dizygous or monozygous twinning (one-tailed test $X_{1}^{2}=0.0004, P=0.49$ ) (Table1). The maternal age at birth could only be determined for 10 sets of twins, and there was no correlation between maternal age and frequency of twinning, al though this may be due to the small sample size. There was no relationship between FMR1 premutation status 


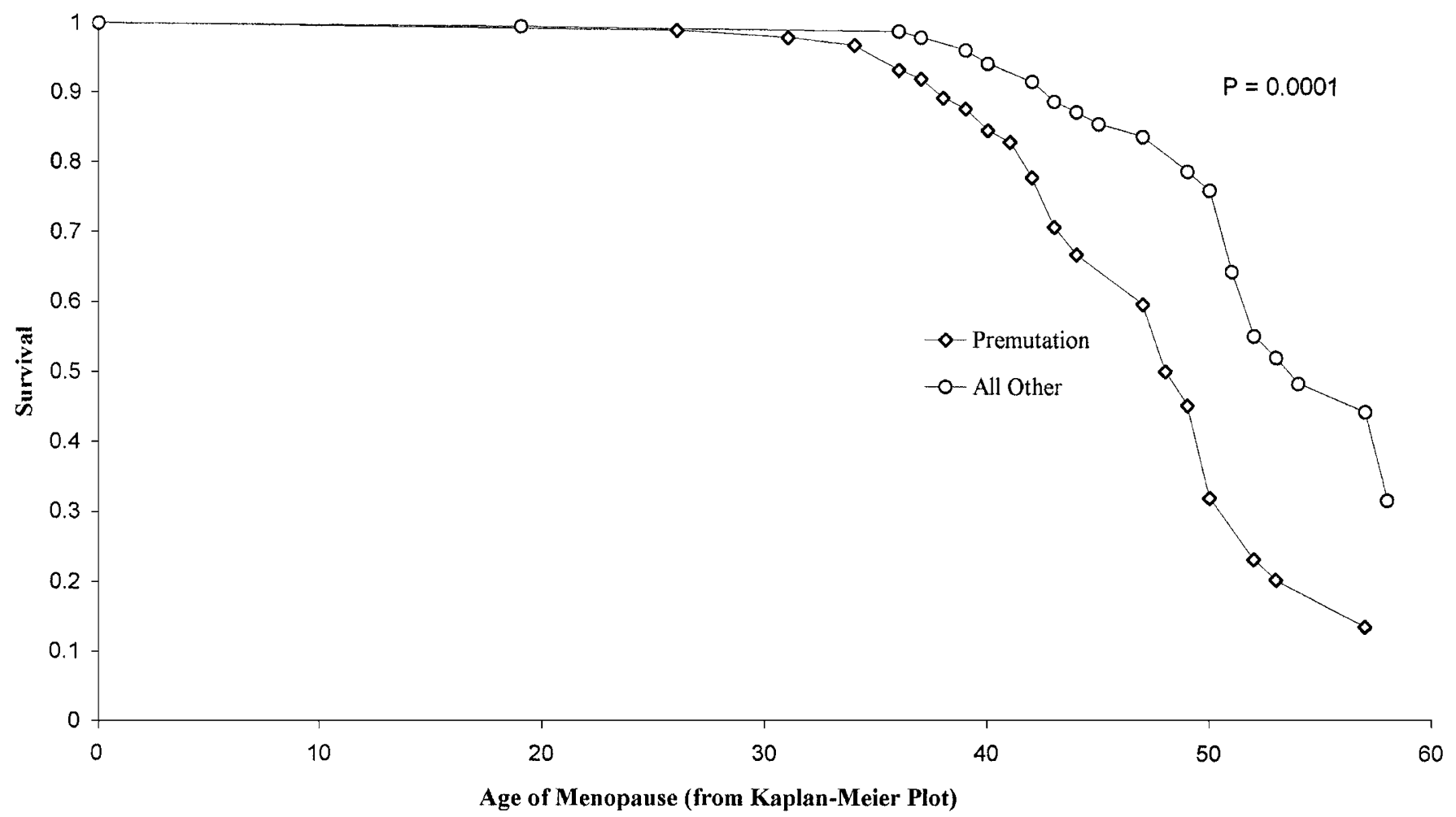

Figure 1 Kaplan-Meier plot of distribution of menopause age as measured by survival analysis, for premutation carriers vs full mutations and controls.

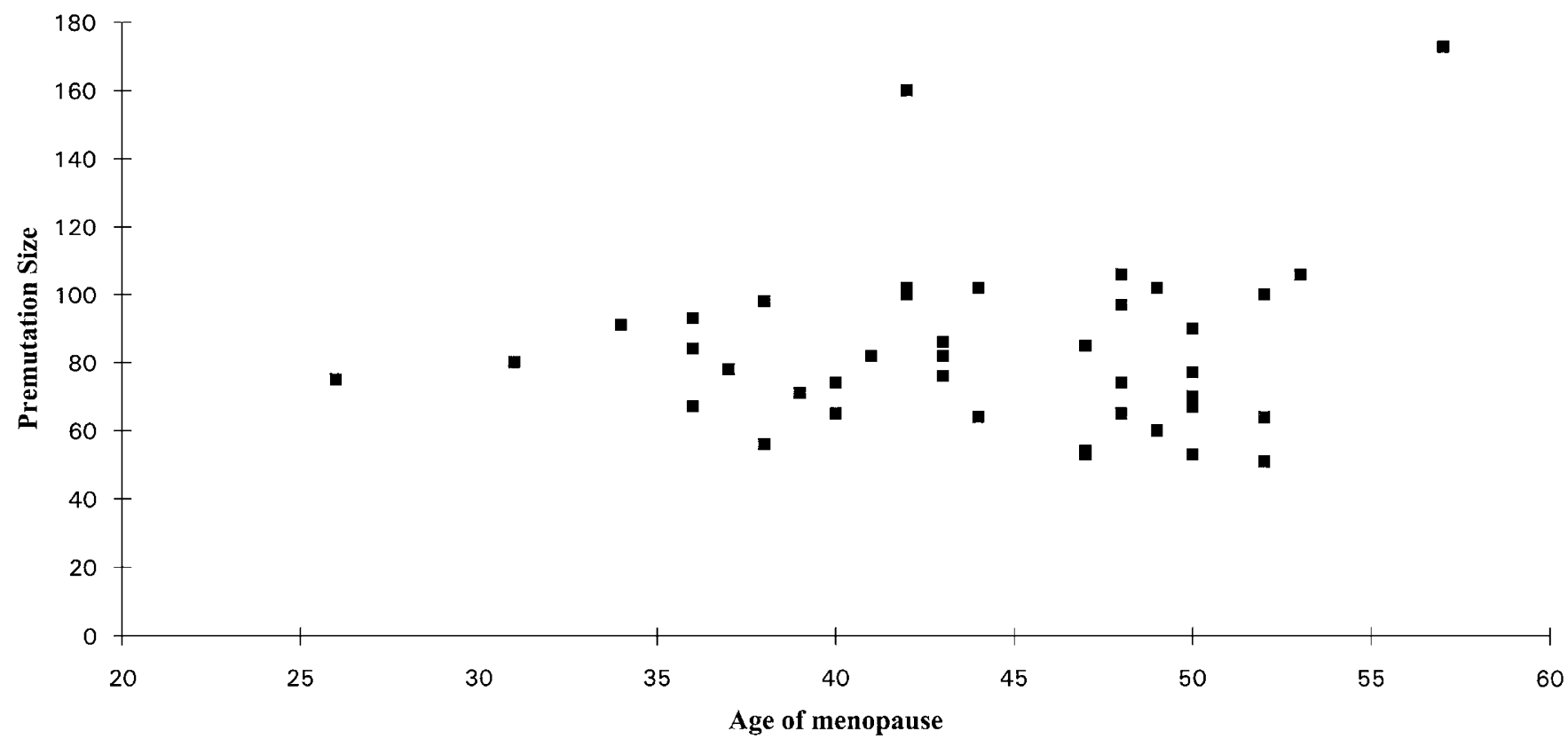

Figure 2 Age of menopause in premutation carriers plotted against the size of the expansion in repeats. Premutations only detected by Southern blot were given an estimate of repeat number. 


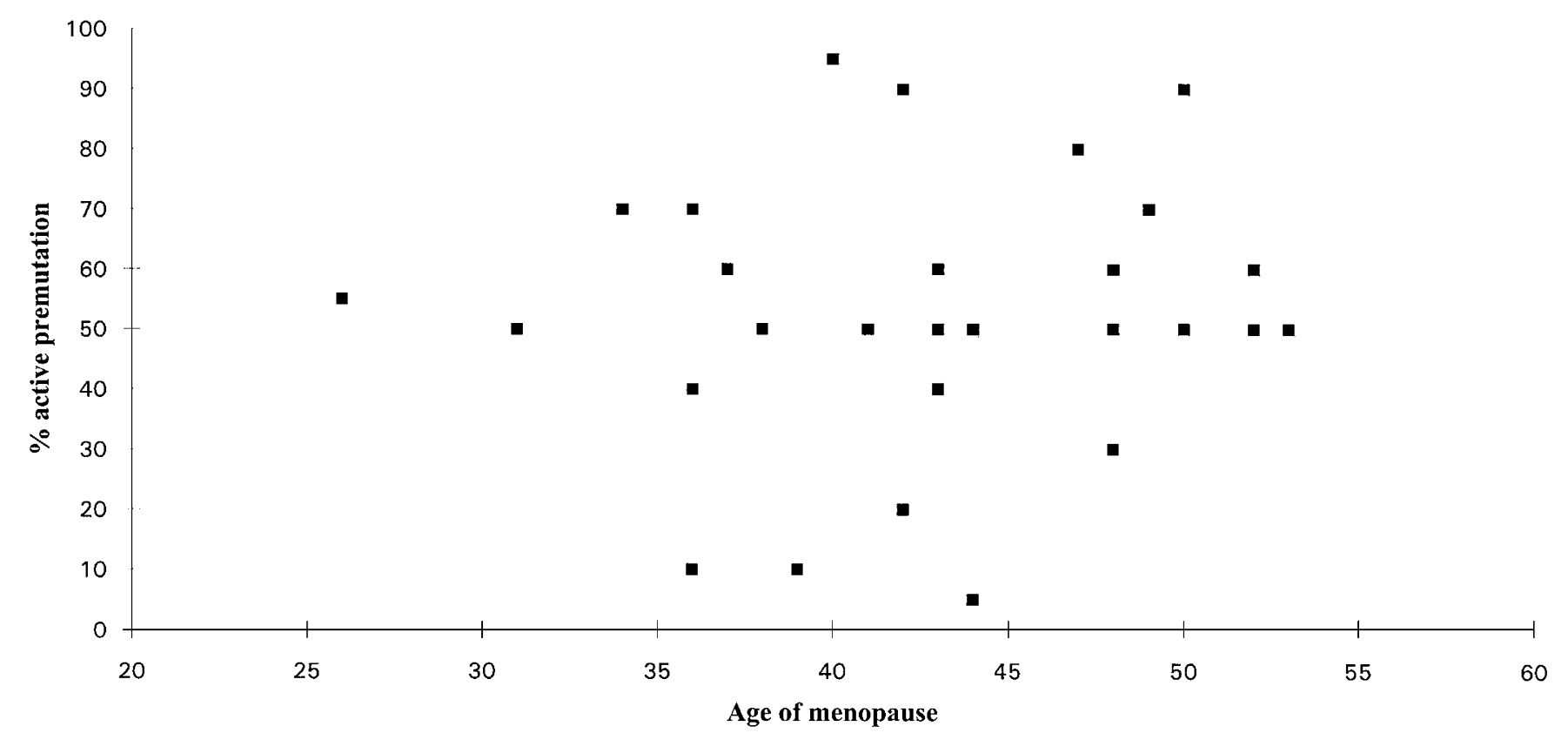

Figure 3 Age of menopause in premutation carriers plotted against the percentage of active $X$ chromosomes carrying the premutation allele, determined by visual estimation.

Table 1 Frequency of twinning

\begin{tabular}{llll}
\hline Mother's repeat category & Monozygous & Dizygous & Unknown \\
\hline Normal $(n=205)$ & 0 & 6 & 8 \\
Premutation $(n=116)$ & 0 & 2 & 1 \\
Full mutation $(n=31)$ & 0 & 1 & 2 \\
\hline
\end{tabular}

Table 2 Pregnancy outcome

\begin{tabular}{lll}
\hline Mother's repeat category & Normal births & Unfavourable outcome \\
\hline Normal $(n=205)$ & 202 & 39 \\
Premutation $(n=116)$ & 210 & 41 \\
Full mutation $(n=31)$ & 49 & 14 \\
\hline
\end{tabular}

and frequency of an unfavourable outcome, either spontaneous abortion, stillbirth or therapeutic abortion (one-tailed test $X_{1}^{2}=0.0007, P=0.49$ ) (Table2).

\section{Hormone analysis}

The results of the analysis of FSH and oestradiol have been published, ${ }^{13}$ but we also tested the two subunits of the hormone inhibin which is thought to be a good predictor of ovarian reserve. Because in hibin levels vary considerably over the follicular phase of the cycle, only sera taken on days 3,4 or 5 were considered in the analysis. There was no correlation between repeat size and inhibin A or B (results not shown).

\section{Discussion}

The association between FRAXA premutations and POF is now well recognised and has been demonstrated in women ascertained through both mental retardation and ovarian failure. ${ }^{6,7}$ However the underlying mechanism is not known. One feature of the association is that not all premutation carriers are affected. In our series only $15 \%$ of the premutation carriers over 40 years of age at interview had their menopause before 40 , and in the larger data series from an international collaborative group the incidence of POF in the similar group was about $24 \% .{ }^{6}$ However premutation carriers with POF may represent the tail of a distribution and in fact all premutation carriers have an earlier menopause than they might otherwise have done. A general reduction in menopause age was reported by Partington et al ${ }^{14}$ who asked women in the UK Fragile $X$ Society when they had their menopause. Unfortunately there is a bias in a study of this type, as women who had an early menopause are more likely to reply to a mailed questionnaire. In addition, control frequencies were quoted from published series rather than matched controls, and women were classified as carriers, with no distinction between premutations and full mutations. We have analysed the relationship between fragile $X$ status and menopause age in our study by performing a survival analysis, allowing the inclusion of women who had not yet had menopause as well as postmenopausal women. This approach had the added advantage of reducing the bias due to variation in the age of interview between the groups. The results of the survival analysis demonstrated a significant shift in menopause age for the entire premutation group when compared with the full mutations and controls. 
These results provide compelling evidence that it is the premutation alone which is having an effect on menopause age and not, as has been suggested, a linked genetic factor. ${ }^{15}$ FMR1 may be morelike many of the other triplet repeat genes than previously supposed. Although the fragile $X$ mental retardation syndrome is undoubtedly caused by inactivation of FMR1, there may be a spectrum of disease phenotypes associated with different allele sizes. A reduction in menopause age is one phenotype that is associated with the premutation category, although there is no evidence from our study that the size of the premutation or the ratio of $X$ inactivation correlates with menopause age. In addition we have reported an excess of intermediate (41-60 repeats) FRAXA alleles in a group of boys with non-specific learning difficulty, suggesting that even relatively small expansions in FMR1 may have a phenotypic effect. ${ }^{16}$

In women who are still cycling we have demonstrated an increased level of serum FSH in the premutation group compared with controls. ${ }^{13}$ In a small pilot study Braat et al ${ }^{17}$ reported the hormonal profiles of nine premutation carriers under age 40 and found levels suggestive of ovarian failure in eight of them. Thus even in the absence of early menopause the premutation group appears to have the hormonal markers of ovarian failure. In the present study we used a relatively new hormonal marker - serum inhibin levels. Inhibin is a hormone with two subunits, $A$ and $B$, and inhibin $B$ is produced by the small antral follicles during the first few days of the cycle and thus is potentially the best marker of ovarian reserve. We did not find any significant differences in serum inhibin A or B levels between the groups. However, it is likely that our study was not rigorous enough to detect the differences in inhibin secretion: since inhibin levels change rapidly on days $3-4$ of the cycle it is advisable to take a second measurement in mid-cycle, when there are no maturing follicles. ${ }^{18}$ The primary aim of the study was to determine menopause age and not to measure inhibin so it was impractical to collect two samples per cycle. Therefore we consider our inhibin data to be inconclusive.

Reports of an excess of dizygous twins in premutation carriers. ${ }^{8,19}$ prompted us to speculate that the effect of a premutation allele may be to induce premature aging of the ovary, as an increase in the rate of dizygous twinning is associated with advanced maternal age and mothers of dizygous twins have an earlier menopause than mothers of monozygous twins. ${ }^{20}$ However, in agreement with Sherman et $a l,{ }^{9}$ we found no evidence of an increase in dizygous twinning in premutation carriers. There were in fact fewer dizygous twins born to premutation carriers than to either of the other groups.

The presumed cause of the increase in dizygous twinning with increasing maternal age is the associated rise in FSH caused by reduction in oocyte number. We have shown that premutation carriers have raised FSH as a group but this did not correlate with an increase in dizygous twins.
As trisomy is very strongly correlated with maternal age and as about $26 \%$ of spontaneous abortions and about $4 \%$ of stillbirths are trisomic, ${ }^{21}$ we determined the frequency of spontaneous abortions, stillbirths, therapeutic abortions (which have a high likelihood of being chromosomally abnormal) and known chromosomally abnormal liveborns in our population. We found no evidence for an excess of trisomic conceptuses in our sample. Presumably the mechanism which causes an increase of non-disjunction in older mothers is not affected by the changes in hormonal profile associated with impending menopause.

In conclusion, FRAXA premutations are associated with premature aging of ovaries since women with premutations have a lower age of menopause and raised serum FSH compared with full mutations and controls. Two other features of an aging ovary, namely an increase in dizygous twinning and trisomy, do not appear to be associated with premutations, suggesting that these features are governed by a separate mechanism.

\section{Acknowledgements}

We are extremely grateful to Professor Patricia Jacobs for advice and discussion, Professor David Baird and Dr Nigel Groome for inhibin testing, and Dr Gerard Conway and Nadia Payne for FSH and oestradiol testing. The work was funded by the Wellcome Trust.

\section{References}

1 Ashley CT, Warren ST: Trinucleotide repeat expansion and human disease. Annu Rev Genet 1995; 29: 703-728.

2 Oberlé I, Rousseau F, Heitz D, Kretz C, Devys D et al: Instability of a 550-base pair DNA segment and abnormal methylation in fragile X syndrome. Science 1991; 252: 1097-1102.

3 Sutherland GR, Mulley JC: Fragile $X$ syndrome and fragile XE mental retardation. Prenat Diagn 1996; 16: 1199-1211.

4 Feng Y, Lakkis L, Devys D, Warren ST: Quantitative comparison of FMRI gene expression in normal and premutation alleles. Am J Hum Genet 1995; 56: 106-113.

5 Schwartz CE, Dean J, Howard-Peebles PN et al: Obstetrical and gynecological complications in Fragile $\mathrm{X}$ carriers: a multicenter study. Am J Med Genet 1994; 51: 400-402.

6 Allingham-Hawkins DJ, Babul-Hirji R, Chitayat D et al: Fragile X premutation is a significant risk factor for premature ovarian failure: the International collaborative POF in fragile $X$ study preliminary data. Am J Med Genet 1999; 83: 322-325.

7 Murray A, Webb J. Grimley S, Conway G, Jacobs P: Studies of FRAXA and FRAXE in women with premature ovarian failure. J Med Genet 1998; 35: 637-641.

8 Turner G, Robinson H, Wake S, Martin N: Dizygous twinning and premature menopause in fragile X syndrome. Lancet 1994; 344: 1500.

9 Sherman SL, Meadows KL, Ashley AE: Examination of factors that influence the expansion of the fragile $X$ mutation in a sample of conceptuses from known carrier females. Am J Med Genet 1996; 64: 256-261.

10 Bulmer MG: The frequency of twins: maternal age and parity. In: The Biology of Twinning in Man. Oxford University Press: London. 1970; 74-83.

11 Groome NP, Illingworth PJ, O'Brien M et al: Detection of dimeric inhibin A throughout the menstrual cycle by a 2-site enzyme immunoassay. Clin Endocrin 1994; 6: 717-723. 
12 Groome NP, Illingworth PJ, O'Brien M et al: Measurement of dimeric inhibin B throughout the menstrual cycle. J Clin Endocrin Metab 1996; 74: 600-607.

13 Murray A, Webb J, MacSwiney F, Shipley EL, Morton NE, Conway GS: Serum concentrations of follicle stimulating hormone may predict premature ovarian failure in FRAXA premutation women. Hum Rep 1999; 14: 1217-1218.

14 Partington MW, Moore DY, Turner GM: Confirmation of early menopause in fragile X carriers. Am J Med Genet 1996; 64: 370-372.

15 Fisch G, Kenneson A, Warren S, Zelterman D: Evidence of linkage disequilibrium between POFI and FRAXAC1. Am J Hum Genet 1998; 63: Suppl Abs1215.

16 Murray A, Youings S, Dennis $N$ et al: Population screening at the FRAXA and FRAXE loci: molecular analyses of boys with learning difficulties and their mothers. Hum Mol Genet 1996; 5: 727-735.
17 Braat DDM, Smits APT, Thomas CMG: Menstrual disorders and endocrine profiles in fragile $X$ carriers prior to 40 years of age: $A$ pilot study. Am J Med Genet 1999; 83: 327-328.

18 Danforth DR, Arbogast LK, Mroueh J et al: Dimeric inhibin: a direct marker of ovarian ageing. Fert Steril 1998; 70: 119-123.

19 Fryns J-P: The female and the fragile X: A study of 144 obligate female carriers. Am J Med Genet 1986; 23: 157-169.

20 Martin NG, Healey SC, Pangan TS, Heath AC, Turner G: Do mothers of dizygotic twins have earlier menopause? A role for fragile X? Am J Med Genet 1997; 69: 114-116.

21 Hassold TJ, Jacobs PA: Trisomy in man. Ann Rev Genet 1984; 18: 69-97. 